\title{
Retinoblastoma Binding Protein 5 Correlates with the Progression in Hepatocellular Carcinoma
}

\author{
Huiling Zhou, ${ }^{1}$ Jingjing Bao, ${ }^{1}$ Xiaowei Zhu, ${ }^{1}$ Guihong Dai, ${ }^{1}$ Xiaoqin Jiang, ${ }^{1}$ Xia Jiao, \\ Haihui Sheng, ${ }^{2}$ Junxing Huang, ${ }^{3}$ and Hong Yu ${ }^{1}{ }^{1}$ \\ ${ }^{1}$ Department of Pathology, Taizhou People's Hospital, Jiangsu Province, China \\ ${ }^{2}$ Shanghai Engineering Center for Molecular Medicine, National Engineering Center for Biochip at Shanghai, Shanghai, China \\ ${ }^{3}$ Department of Oncology, Taizhou People's Hospital, Taizhou, Jiangsu Province, China \\ Correspondence should be addressed to Hong Yu; yuhongmiaomiao@163.com
}

Received 26 June 2018; Revised 16 September 2018; Accepted 24 September 2018; Published 7 November 2018

Academic Editor: Robert J. Lee

Copyright (C) 2018 Huiling Zhou et al. This is an open access article distributed under the Creative Commons Attribution License, which permits unrestricted use, distribution, and reproduction in any medium, provided the original work is properly cited.

\begin{abstract}
Hepatocellular carcinoma (HCC) is one of the most common malignancy tumors with insidious onset, rapid development and metastasis, and poor prognosis. Therefore, it is necessary to understand molecular mechanisms of HCC and identify clinically useful biomarkers for it. This study aimed to investigate the role of retinoblastoma binding protein 5 (RBBP5) in HCC. The expression level of RBBP5 was examined by immunohistochemistry and western blot. The effect of RBBP5 on cell cycle, proliferation, apoptosis, and drug sensitivity was analyzed. RBBP5 was significantly upregulated in HCC tissues and cells. High RBBP5 expression was significantly associated with elevated level of AFP, advanced TNM stage, high Ki-67 expression, larger tumor size, and poor prognosis. Knockdown of RBBP5 significantly inhibited proliferation of HCC cells through cell cycle arrest. In addition, inhibition of RBBP5 increased the sensitivity of HCC cells to doxorubicin. In conclusion, our findings suggest that RBBP5 plays an important role in the progression of HCC and may serve as a novel biomarker and potential therapeutic target for HCC.
\end{abstract}

\section{Introduction}

Hepatocellular carcinoma (HCC) is the sixth most common cancer and the third most common cause of cancer-related death worldwide with insidious onset, rapid development and metastasis, and poor prognosis $[1,2]$. HCC remains a major threat to public health around the world, especially in China. Therefore, the early diagnosis and treatment of HCC is critical to improving patient outcomes. With the progress of molecular biology, it is generally accepted that multiple signaling pathways such as MAPK, PI3K/AKT, and Wnt signaling pathways are the key mechanisms leading to formation of HCC $[3,4]$. Since abnormal cell cycle regulation plays an important role in carcinogenesis, investigation of the mechanism of cell cycle regulation may help identify potential biomarkers and therapeutic targets for HCC.

Retinoblastoma binding protein 5 (RBBP5) was determined as the binding protein of $\mathrm{RB}$ transcriptional corepressor 1 (RB1) which is one of the best studied tumor suppressor proteins [5]. RBBP5 binds to underphosphorylated pRB1 in the regulation of cell cycle by RB1 pathway [6-8]. RBBP5 has been reported to be involved in a variety of tumors [9]. RBBP5 regulates DNA-damaging agent-induced apoptosis in tumor cells. In glioma, RBBP5 was highly expressed, participated in G1-S transition, and was also associated with the inhibition of apoptosis [10]. Furthermore, RBBP5 is a core member of MLL/SET (mixed lineage leukemia/set-domain containing) complexes involved in tumor cell cycle progression through an MLL-E2F axis which controls the expression of cyclins E, $\mathrm{A}$, and $\mathrm{B}$ [11-13]. RBBP5 is required for $\mathrm{H} 3 \mathrm{~K} 4$ methylation, which is a common marker of transcriptional activity in tumors, such as leukemia $[11,14]$. In addition, many other RB1 interacting proteins or their cognate proteins such as SYF2 and Bog are involved in the HCC process $[15,16]$. However, the expression and precise role of RBBP5 in HCC remains virtually unknown.

In the present study, we examined the expression level of RBBP5 in HCC and adjacent noncancerous tissues, and the 
TABLE 1: Correlation of RBBP5 expression with clinicopathological factors in 94 HCC patients.

\begin{tabular}{|c|c|c|c|}
\hline \multirow{2}{*}{ Clinicopathological factors } & \multicolumn{2}{|c|}{ RBBP5 expression } & \multirow{2}{*}{$P$ Value } \\
\hline & Low & High & \\
\hline \multicolumn{4}{|l|}{ Sex } \\
\hline female & 10 & 8 & 0.185 \\
\hline male & 28 & 48 & \\
\hline \multicolumn{4}{|l|}{ Age (years) } \\
\hline$<45$ & 17 & 18 & 0.278 \\
\hline$\geq 45$ & 21 & 38 & \\
\hline \multicolumn{4}{|l|}{ HbsAg } \\
\hline negative & 11 & 13 & 0.631 \\
\hline positive & 27 & 43 & \\
\hline \multicolumn{4}{|l|}{$\operatorname{AFP}(\mathrm{ng} / \mathrm{ml})$} \\
\hline$<50$ & 21 & 17 & 0.019 \\
\hline$\geq 50$ & 17 & 39 & \\
\hline \multicolumn{4}{|l|}{ Cirrhosis } \\
\hline negative & 19 & 22 & 0.397 \\
\hline positive & 19 & 34 & \\
\hline \multicolumn{4}{|l|}{ AJCC stage } \\
\hline I-II & 28 & 27 & 0.019 \\
\hline III-IV & 10 & 29 & \\
\hline \multicolumn{4}{|l|}{ Tumor size $(\mathrm{cm})$} \\
\hline$<5$ & 24 & 20 & 0.012 \\
\hline$\geq 5$ & 14 & 36 & \\
\hline \multicolumn{4}{|l|}{ No. of tumor nodes } \\
\hline single & 24 & 24 & 0.062 \\
\hline multiple & 14 & 32 & \\
\hline \multicolumn{4}{|l|}{ Capsular formation } \\
\hline negative & 10 & 26 & 0.055 \\
\hline positive & 28 & 30 & \\
\hline \multicolumn{4}{|l|}{ Metastasis } \\
\hline negative & 31 & 47 & 0.786 \\
\hline positive & 7 & 9 & \\
\hline \multicolumn{4}{|l|}{ Vein invasion } \\
\hline negative & 27 & 37 & 0.658 \\
\hline positive & 11 & 19 & \\
\hline \multicolumn{4}{|l|}{ Ki-67 expression } \\
\hline low & 34 & 2 & $<0.001$ \\
\hline high & 4 & 54 & \\
\hline
\end{tabular}

correlation between its expression and clinicopathological parameters. Furthermore, we investigated the effect of knockdown of RBBP5 on the proliferation, cell cycle, apoptosis, colon formation, and drug sensitivity of HCC cells. RBBP5 exhibits potential as a prognostic biomarker and therapeutic target for HCC.

\section{Materials and Methods}

2.1. Patients and Tissue Samples. A total of 94 pairs of HCC and paracancerous tissues were obtained from patients who underwent hepatic surgical resection without preoperative systemic chemotherapy at Taizhou People's Hospital between 2007 and 2010. The clinicopathological characteristics of the patients were listed in Table 1 . These 94 patients whose average age was 49.0 years (range, 23-74) comprised 76 males and 18 females. In addition, $41.5 \%$ of patients were at TNM stages III and IV according to the American Joint Committee on Cancer (AJCC) TNM stage. The follow-up time duration was 5 years, with a range of 1-60 months. Furthermore, additional 8 pairs of fresh HCC and paracancerous tissues were collected for western blot. We obtained the written informed consent from every patient, and the study was approved by the Ethics Committee of Taizhou People's Hospital.

2.2. Immunohistochemistry (IHC) and Scoring. The sections were deparaffinized in xylene and rehydrated through graded alcohol. Immunoreactivity was enhanced following antigen 
retrieved by high temperature and pressure. Endogenous peroxidase activity was blocked with $3 \%$ hydrogen peroxide in PBS. After being rinsed in phosphate-buffered saline (PBS, $\mathrm{pH} 7.2$ ), $10 \%$ goat serum was used for $1 \mathrm{~h}$ at room temperature to block any nonspecific reactions. The slides were incubated with Anti-RBBP5 antibody (dilution 1:200, Sigma-Aldrich, MO, USA) overnight at $4^{\circ} \mathrm{C}$ and anti-Ki-67 antibody (dilution 1:500, Millipore, Bedford, MA, USA) at room temperature for $2 \mathrm{~h}$ and then incubated with the secondary antibody at room temperature for $1 \mathrm{~h}$. The slides were then counterstained with $3,3^{\prime}$-diaminobenzidine (DAB) and 20\% hematoxylin. Finally, the slides were examined under a light microscope (Leica Microsystems, Wetzlar, Germany).

All immunostained slides were assessed in a blinded manner by two pathologists without knowledge of any clinicopathological information. Immunostaining score was calculated for each section according to the proportion of stained tumor cells and the intensity of the staining [17]. The intensity of staining was scored as 0 (no staining), 1 (weakly staining), 2 (moderately staining), or 3 (strongly staining). According to the percentage of positive tumor cells, the extent of staining was scored as 0 ( $\leq 10 \%), 1$ (11-30\%), $2(31-50 \%), 3(51-70 \%)$, and $4(\geq 70 \%)$. These two scores were multiplied into a final score $(0-12)$ for each tissue. Samples were classified as low expression $($ score $\leq 3)$ or high expression (score $>3$ ).

2.3. Western Blot. Cells and tissues were immediately resuspended in a homogenization buffer $(50 \mathrm{mM}$ Tris. $\mathrm{HCl} \mathrm{pH}$ 7.5, $150 \mathrm{mM} \mathrm{NaCl}, 1 \% \mathrm{NP}-40,1 \%$ sodium deoxycholate, $0.1 \%$ SDS, $1 \mathrm{mM}$ EDTA) containing Complete Protease Inhibitor Cocktail (Roche Diagnostics, Mannheim, Germany), and then centrifuged at $12,000 \mathrm{rpm}$ for $30 \mathrm{~min}$ at $4^{\circ} \mathrm{C}$ to collect the supernatant liquid. Total protein concentration was determined using a Bio-Rad protein assay (Bio-Rad, Hercules, CA, USA). The supernatant was diluted in $2 \times$ sodium dodecyl sulfate (SDS) loading buffer and boiled for $15 \mathrm{~min}$. Subsequently, the samples were subjected to $10 \%$ SDS-polyacrylamide gel electrophoresis (SDS-PAGE) separation and then transferred to polyvinylidene difluoride filter (PVDF) membranes (Millipore, Bedford, MA, USA). After the membranes were blocked with 5\% dried skim milk in TBST (20 mM Tris, $150 \mathrm{mM} \mathrm{NaCl}, 0.05 \%$ Tween-20) for $2 \mathrm{~h}$, they were incubated with primary antibodies overnight at $4^{\circ} \mathrm{C}$. The membrane was washed with TBST three times for $5 \mathrm{~min}$ each, and then horseradish peroxidase-linked IgG (Pierce Biotechnology, Rockford, IL, USA) was added to the membrane as the second antibody at a dilution of 1:5000 according to the manufacturer's instructions. The immune complexes were visualized by chemiluminescence (NEN Life Science Products, Boston, MA, USA).

2.4. Cell line and Cell Culture. The human HCC cell lines (Huh7, Hep3B, HepG2, and SMCC-7721) and L02 normal hepatocytes were purchased from Cell Bank of Type Culture Collection of Shanghai Institute of Cell Biology, Chinese Academy of Sciences. (Shanghai, China). All cells were cultured in high-glucose Dulbecco's modified Eagle's medium (DMEM) (Sigma, St. Louis, MO, USA) with 10\% fetal bovine serum (FBS) (HyClone, Logan, UT) and $100 \mathrm{U} / \mathrm{ml}$ penicillin-streptomycin mixture and in a $37^{\circ} \mathrm{C}$ incubator with $5 \% \mathrm{CO}_{2}$.

2.5. SiRNA Synthesis and Transfection. Control siRNA and RBBP5 siRNA oligos were synthesized by GenePharma (Suzhou, China). The sequences of RBBP5-targeting siRNA were $5^{\prime}$-GCA AUA CCA CAG CCA UUA ATT UUA AUG GCU GUG GUA UUG CTT-3' (siRBBP5-3-1); $5^{\prime}$-CCC UGU ACA UCU GGG AGA ATT UUC UCC CAG AUG UAC AGG GTT-3' (siRBBP5-3-2); 5' -GCA CCA GAC UUC AAA GAA UTT AUU CUU UGA AGU CUG GUG CTT-3' (siRBBP5-3-3). Cell transfection assays were performed with Lipofectamine 2000 transfection reagent (Invitrogen, CA, USA) according to manufacturer's protocol.

2.6. Cell Cycle Analysis. For cell cycle analysis, cells were harvested at a proper time and washed twice with ice-cold PBS and then fixed with $70 \%$ ethanol for $24 \mathrm{~h}$ at $4^{\circ} \mathrm{C}$. Followed by wash with PBS three times, the cells were resuspended in PBS containing RNase A $(100 \mu \mathrm{g} / \mathrm{mL})$ and propidium iodide $(100 \mu \mathrm{g} / \mathrm{mL})$ and then incubated at $37^{\circ} \mathrm{C}$ for $30 \mathrm{~min}$. Cell cycle distribution was analyzed by BD FACSCalibur flow cytometer (BD Biosciences, CA, USA).

2.7. Cell Proliferation Assay. The cells were inoculated at a density of $2 \times 10^{4}$ /well into 96-well plate (Corning Inc., Corning NY, USA) and incubated for $24 \mathrm{~h}$. Cell Counting Kit8 reagents (Dojindo, Kumamoto, Japan) were added to each well at due time, and the cells were incubated for an additional $2 \mathrm{~h}$ at $37^{\circ} \mathrm{C}$. The absorbance at the wavelength of $490 \mathrm{~nm}$ was measured with a microplate reader (Bio-Rad, Hercules, CA, USA).

Colony Formation Assay. Cells were inoculated at a density of 200 cells/well into 6-well plates and followed by transfection with control and siRBBP5 transfection according to the manufacturer's instructions. We analyzed the clearly visible colonies (C50 cells/colony) using $0.5 \%$ crystal violet stain for 30 min after 14 days of culture.

Apoptosis Detection. The cells transfected with siRBBP5 and control cultured for $48 \mathrm{~h}$ and were harvested. Apoptosis was assayed using BD FACSCalibur flow cytometer (BD Biosciences, CA, USA) with Annexin V-FITC apoptosis detection kit (Dojindo, Kumamoto, Japan) according to the manufacturer's instructions.

2.8. Drug Sensitivity Assay. The cells were inoculated at a density of $2 \times 10^{4} /$ well into 96 -well plate and incubated for $24 \mathrm{~h}$ and then were suspended in DMEM containing $10 \%$ (FBS). Cells were treated with different dose of doxorubicin (DOX) 48 hours. The viability of the cells was examined by CCK-8 assay.

2.9. Statistical Analyses. All experiments were performed in triplicate. Quantitative variables were expressed as mean \pm standard deviation and analyzed using Student's t-test. The $\chi^{2}$ test was used to analyze the association between RBBP5 

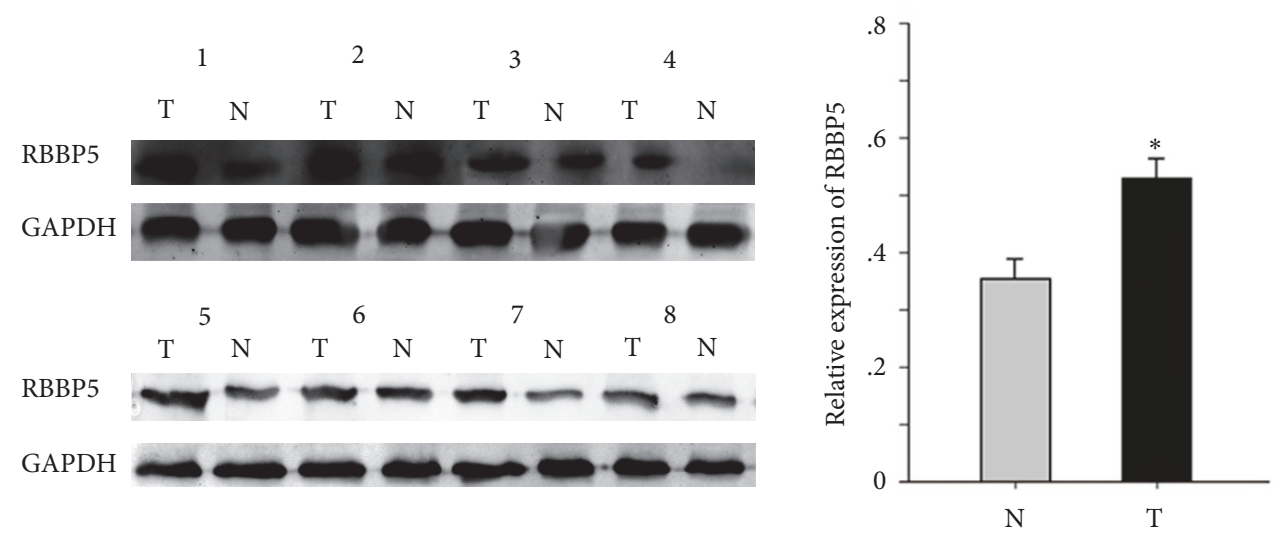

(a)
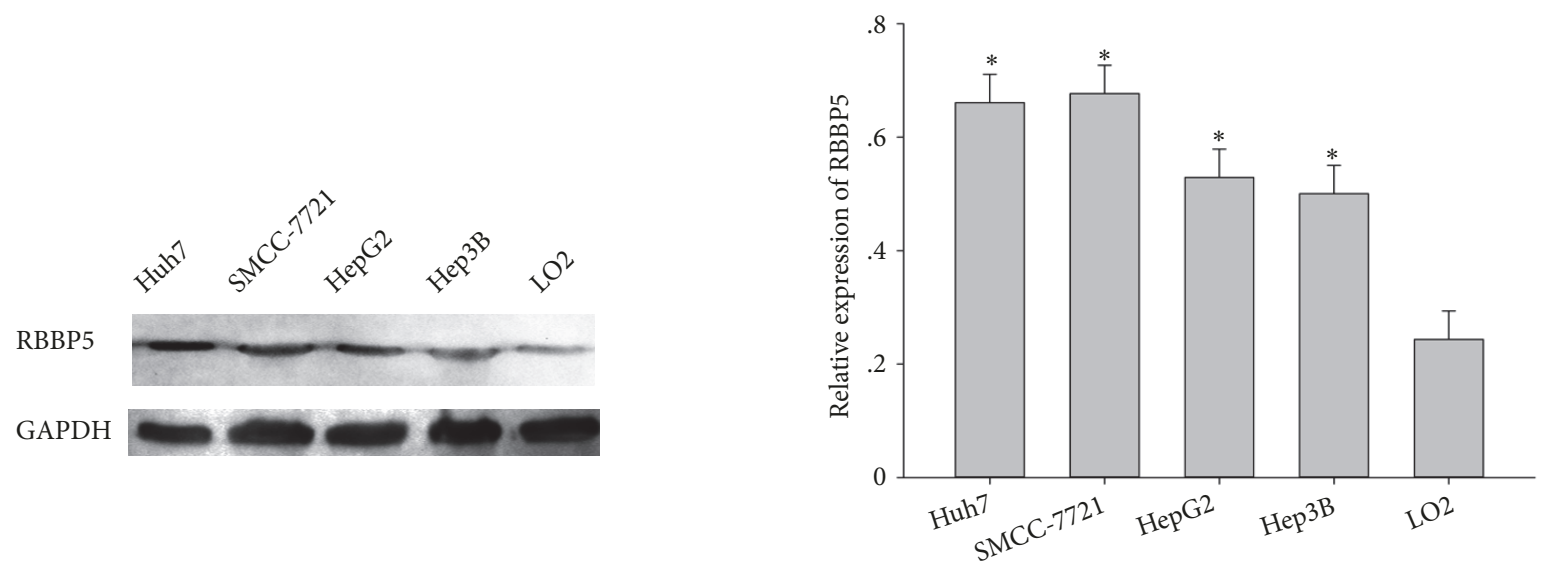

(b)

FIgURE 1: The expression level of RBBP5 in HCC tissues and cell lines. (a) Western blot analysis showed that the expression levels of RBBP5 in HCC tissues (T) were significantly higher than those in adjacent noncancerous tissues (N). (b) RBBP5 expression was upregulated in HCC cells compared with the normal liver cell line (L02). GAPDH was used as a control for protein load and integrity. All experiments were performed in triplicate. $* P<0.05$.

and Ki-67 expression and the clinicopathological features. Survival analysis was performed by using the Kaplan-Meier method and the Log-rank test. The Cox's proportional hazards model was used to identify the factors related to prognosis through a multivariate survival analysis. A $P$ value $<0.05$ was considered statistically significant. All statistical analyses were performed with the SPSS 21.0 statistical analysis software (SPSS Inc., Chicago, IL, USA).

\section{Results}

3.1. RBBP5 Was Upregulated in HCC Tissues and Cells. To explore the function of RBBP5 in HCC, we first carried out western blot to examine the expression levels of RBBP5 in HCC tissues and cells. The expression levels of RBBP5 in HCC tissues were significantly higher than those in paracancerous tissues (Figure 1(a)). In addition, high expression level of RBBP5 was confirmed in 4 HCC cell lines (Huh7, Hep3B, HepG2, and SMCC-7721) in comparison with a normal hepatocyte cell line (LO2). These findings implicated that
RBBP5 was upregulated in HCC tissues and cell lines and might be an important oncogenic factor in HCC.

3.2. Clinical Significance of RBBP5 in HCC. We further investigated RBBP5 expression using IHC in 94 pairs of HCC and paracancerous tissues and evaluated its clinical significance. RBBP5 was significantly upregulated in HCC tissues compared with that in adjacent normal tissues $(P=$ 0.013, Figure 2). High RBBP5 expression was significantly correlated with high serum level of $\operatorname{AFP}(P=0.019)$, advanced TNM stage $(P=0.019)$, larger tumor size $(P=0.012)$, and high Ki-67 expression $(P<0.001)$ (Table 1$)$. However, no association was observed between RBBP5 expression and other clinicopathological factors, including sex, age, HbsAg, and cirrhosis. In addition, patients with high RBBP5 expression had shorter survival time than those with low RBBP5 expression $(P<0.001$, Figure 2$)$. Multivariate analysis using the Cox proportional hazards model showed that high RBBP5 expression was an independent factor for prediction of poor outcome in HCC patients $(P<0.001$, Table 2$)$ 

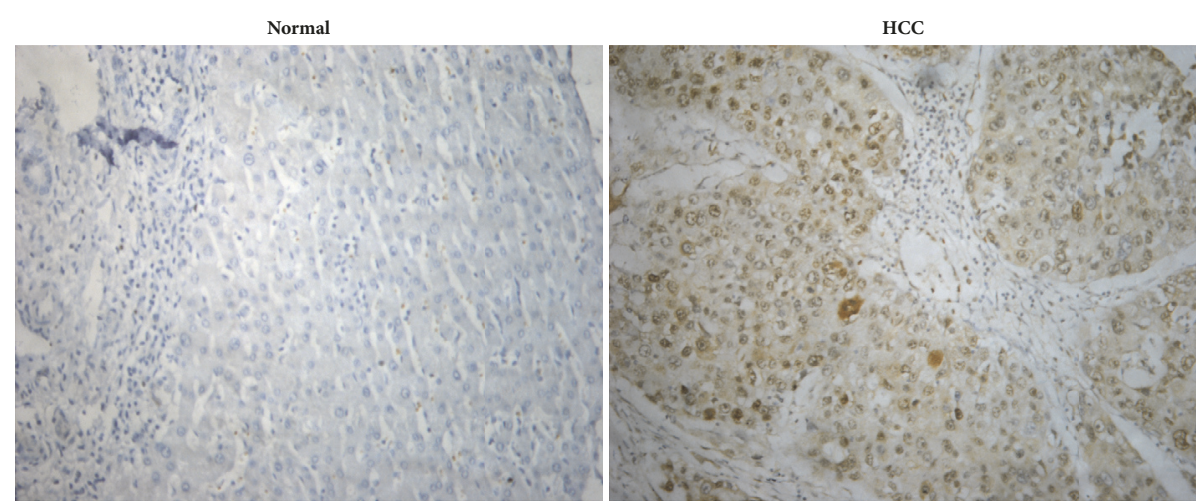

(a)

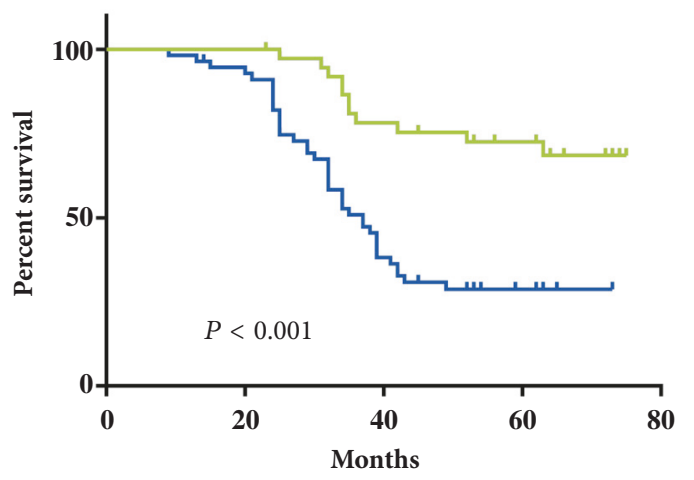

- low RBBP5 expression

十 high RBBP5 expression

(b)

FIGURE 2: Kaplan-Meier estimates of overall survival for 94 HCC patients according to RBBP5 expression. (a) RBBP5 was significantly overexpressed in HCC tissues ( $\mathrm{SP} \times 400)$ compared with the adjacent noncancerous tissues $(\mathrm{SP} \times 400)$. All patients were divided into high (n $=56)$ and low RBBP5 expression groups $(\mathrm{n}=38)$ according to the score. (b) Patients with high RBBP5 expression had shorter survival times than those with low RBBP5 expression (median survival time: 42.9 months $v s .63 .7$ months, $P<0.001$ ).

3.3. Knockdown of RBBP5 Affects Cell Cycle, Proliferation, and Apoptosis of HCC Cells. To further investigate the biological function of RBBP5 in HCC, SMCC-7721 and Huh7 cells with the highest level of RBBP5 were selected for further assays. The expression level of RBBP5 was significantly downregulated in SMCC-7721 and Huh7 cells after transfected with siRBBP5 $(P<0.05$, Figure 3(a)). Not surprisingly, HCC cells were arrested in G1 phase after inhibiting RBBP5 expression $(P<0.05$, Figure $3(\mathrm{~b}))$. The expression levels of cyclin E and proliferating cell nuclear antigen (PCNA) were obviously decreased after knockdown of RBBP5 (Figure 3(a)). These results indicate that RBBP5 plays an important role in cell cycle regulation. In addition, CCK- 8 assays revealed that knockdown of RBBP5 significantly inhibited cell proliferation in SMCC-7721 and Huh7 cells (Figure 3(c)).

3.4. Knockdown of RBBP5 Sensitizes HCC Cells to Doxorubicin. DOX affected the growth of HCC cells in a dose- and time-dependent manner (Figure 4(a)). We further examined whether RBBP5 influenced the sensitivity of HCC cells to doxorubicin. SMCC-7721 and Huh7 cells were collected for growth assay and apoptosis analysis after exposure to DOX for $48 \mathrm{~h}$. The growth of HCC cells was decreased, whereas apoptotic rate was increased after knockdown of RBBP5
(Figures 4(b) and 4(c)). Western blot revealed that the level of cleaved caspase- 3 was significantly increased after depletion of RBBP5 and the DOX addition (Figure 4(d)). Furthermore, knockdown of RBBP5 significantly suppressed the colony formation of SMCC-7721 and Huh7 cells (Figure 4(e)). These results indicate that inhibition of RBBP5 could increase the sensitivity of HCC cells to DOX.

\section{Discussion}

HCC is a complex disease with high metastasis, recurrence, and chemoresistance despite improvement in HCC diagnosis and therapy [18]. Since its multiple molecular mechanisms have not yet been fully elucidated, the long-term survival of HCC patients is far from unsatisfactory. Therefore, the identification of effective therapeutic targets and biomarkers is a great concern in the field of HCC research. RBBP5, also defined as a binding protein of retinoblastoma, is one of the best studied tumor suppressors [6]. However, the role of RBBP5 in HCC carcinogenesis remains virtually obscure.

Previous studies have shown that RBBP5 is upregulated in some types of human cancers including glioma [10] and multiple myeloma [19]. Overexpression of RBBP5 


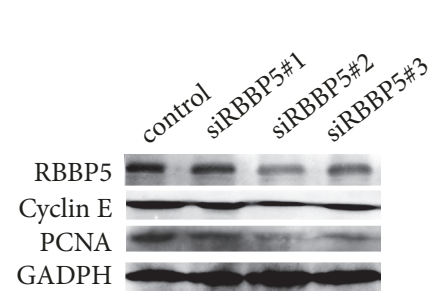

SMCC-7721
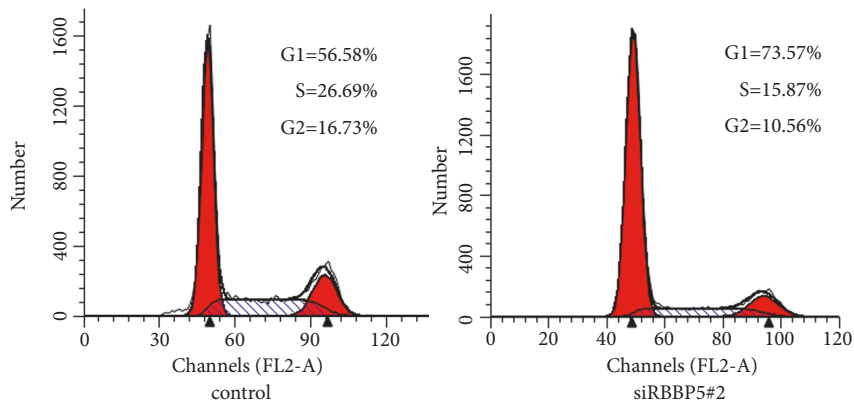

SMCC-7721

(b)

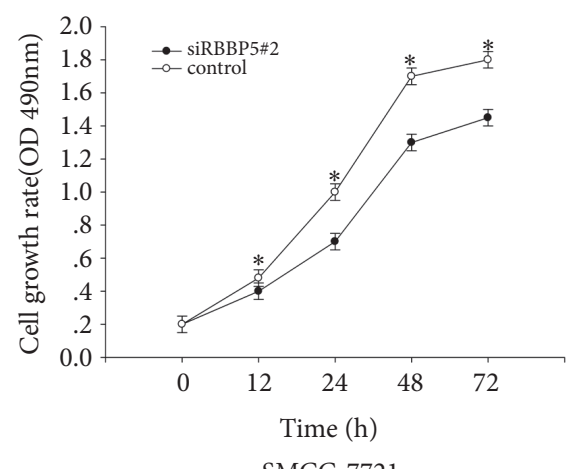

SMCC-7721

(a)
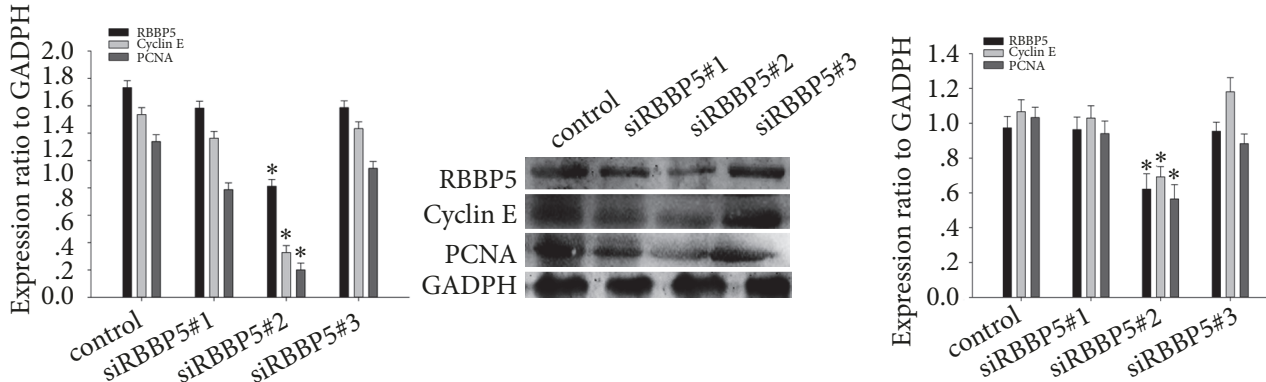

Huh7
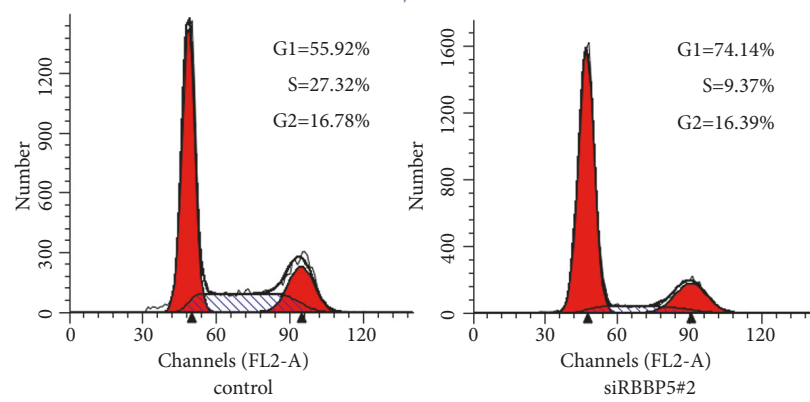

Huh7

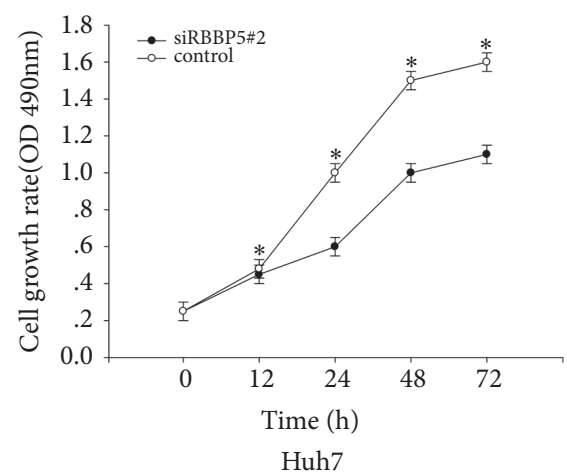

(c)

FIGURE 3: Knockdown of RBBP5 inhibits cell cycle progress and proliferation of HCC cells. (a) Western blot analysis showed that siRBBP5\#2 displayed the most significant knockdown effect on RBBP5 expression and were selected for further experiment. After knockdown of RBBP5 by siRBBP5\#2, Cyclin E and PCNA were significantly downregulated. (b) Depletion of RBBP5 inhibited cell cycle progression of SMCC7721 and Huh7 cells. (c) CCK-8 assay showed that knockdown of RBBP5 inhibited proliferation progress of SMCC-7721 and Huh7 cells. All experiments were performed in triplicate. $* P<0.05$.

promotes cell cycle progression and proliferation and induces chemotherapy resistance of cancer cells [10,19]. In the present study, we found that RBBP5 was significantly upregulated in HCC tissues and cell lines. High RBBP5 expression was associated with aggressive behavior of HCC. RBBP5 was an independent prognostic indicator of survival of HCC patients, which was in agreement with previous study that glioma patients with high RBBP5 expression had worse prognosis [10]. Knockdown of RBBP5 induces cell cycle at G1/S phase and apoptosis and inhibits proliferation in HCC cells. The process may be regulated by direct or indirect stimulation of PCNA and Cyclins. Low RBBP5 expression inhibits cell cycle progression in the process of HCC cell proliferation. Furthermore, inhibition of RBBP5 expression was found to enhance the sensitivity of HCC cells to DOX. These results indicate that RBBP5 plays an important role in the progression of HCC and may be a potential therapeutic target for HCC. However, Liu et al. [19] reported that downregulation of RBBP5 reduced sensitivity to bortezomib and mitoxantrone in RPMI 8226 and NCI-H929 myeloma cell lines adherent to bone marrow stromal cells, indicated that RBBP5 might be the target of bortezomib and mitoxantrone. These results indicate that RBBP5 seems to play different role in the anticancer effect of different drugs. 
TABLE 2: Univariate and multivariate Cox regression analysis of overall survival in 94 HCC patients.

\begin{tabular}{|c|c|c|c|c|}
\hline \multirow{2}{*}{ Clinicopathological factor } & \multicolumn{2}{|c|}{ Univariate analysis } & \multicolumn{2}{|c|}{ Multivariate analysis } \\
\hline & $\mathrm{HR}(95 \% \mathrm{CI})$ & $P$ value & $\operatorname{HR}(95 \% \mathrm{CI})$ & $P$ value \\
\hline Sex, male vs female & $0.999(0.485-2.058)$ & 0.999 & & \\
\hline Age (years), $<45$ vs $\geq 45$ & $1.215(0.669-2.205)$ & 0.522 & & \\
\hline HbsAg, positive vs negative & $0.738(0.403-1.351)$ & 0.324 & & \\
\hline $\operatorname{AFP}(\mathrm{ng} / \mathrm{ml}), \geq 50$ vs $<50$ & $1.339(0.751-2.389)$ & 0.322 & & \\
\hline Cirrhosis, positive vs negative & $1.811(1.035-3.170)$ & 0.038 & $1359(0.705-2.619)$ & 0.360 \\
\hline AJCC stage, III+IV vs I+II & $1.793(1.026-3.132)$ & 0.040 & $1.326(0.739-2.379)$ & 0.344 \\
\hline Tumor size $(\mathrm{cm}), \geq 5$ vs $<5$ & $1.126(0.644-1.969)$ & 0.676 & & \\
\hline No. of tumor nodes, multiple vs single & $2.845(1.563-5.180)$ & 0.001 & $2.417(1.202-4.860)$ & 0.013 \\
\hline Capsular formation, positive vs negative & $0.730(0.416-1.282)$ & 0.273 & & \\
\hline Metastasis, positive vs negative & $1.539(0.786-3.014)$ & 0.209 & & \\
\hline Vein invasion, positive vs negative & $1.366(0.772-2.419)$ & 0.284 & & \\
\hline RBBP5 expression, high vs low & $3.706(1.880-7.307)$ & $<0.001$ & $10.631(3.089-36.592)$ & $<0.001$ \\
\hline Ki-67 expression, high vs low & $2.152(1.139-4.065)$ & 0.018 & $4.072(1.227-13.522)$ & 0.022 \\
\hline
\end{tabular}

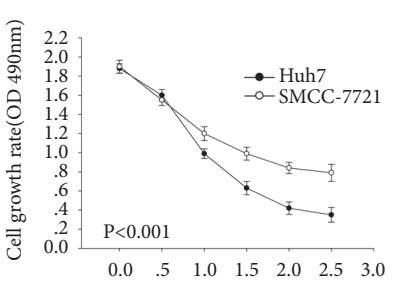

Concentration of DOX $(\mu \mathrm{mol} / \mathrm{L})$

(a)

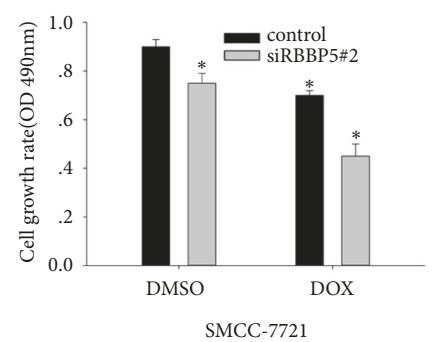

(b)
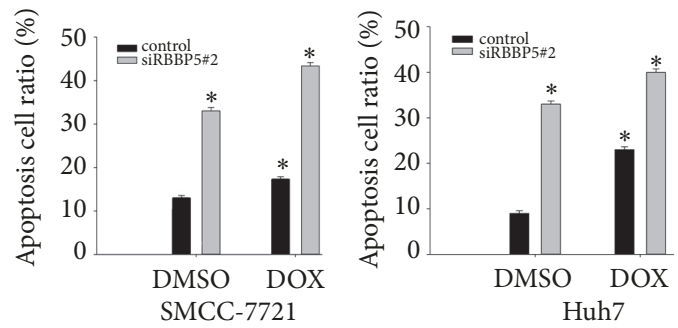

(c)

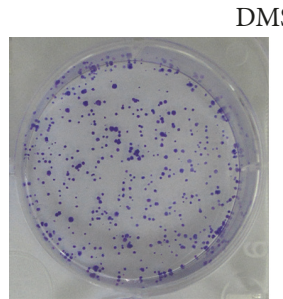

control
DMSO

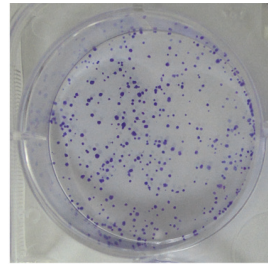

siRBBP5\#2

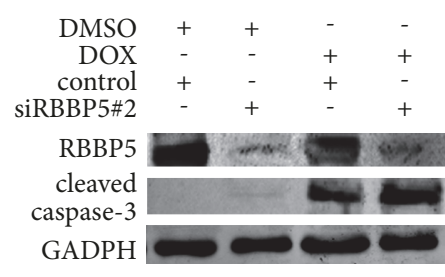

SMCC-7721

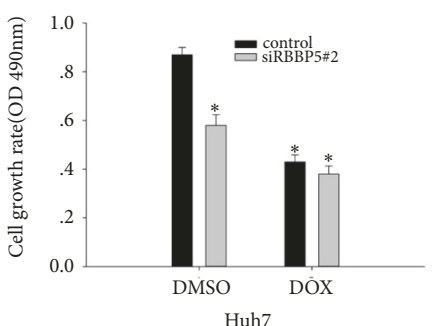

Hub7
0.360

.013

0.022 
RBBP5 is also one of the core components of mixed lineage leukemia 1 (MLL1), a histone 3 lysine 4 (H3K4) methyltransferase complex, and is necessary for H3K4 methyltransferase activity[20, 21]. Furthermore, the $\beta$-propeller domain of RBBP5 has a feature rich surface that can bind nucleic acids and acts as a platform for the recruitment of the MLL complexes to chromatin features or to specific genes [22]. MLL1 is one of the key transcription factors and regulates $\sim 5 \%$ of actively transcribed genes [23]. Dysfunction of MLL1 has been reported to be associated with various cancers such as acute leukemia [24, 25]. It has been demonstrated that MLL1 participates in the cell cycle progression [25]. Dysregulation of the cell cycle can lead to uncontrolled cell proliferation and therefore promote tumor development. In this study, we also found that knockdown of RBBP5 inhibited cell cycle and proliferation and promoted apoptosis of HCC cells. These results indicate that RBBP5 may contribute to HCC development by stimulating cell proliferation and inhibiting HCC apoptosis. However, further studies are required to clarify the underlying mechanism in the development and progression of HCC.

In conclusion, our study provided the first evidence that RBBP5 was conspicuously overexpressed in HCC and was associated with Ki-67 expression, AFP, TNM stage, tumor size, and poor prognosis. In addition, knockdown of RBBP5 can suppress the cell cycle and proliferation, induce apoptosis of HCC cells, and increase its sensitivity to DOX. RBBP5 may serve as a novel biomarker and therapeutic target for HCC.

\section{Data Availability}

The data used to support the findings of this study are included within the article.

\section{Conflicts of Interest}

The authors declare that there are no conflicts of interest regarding the publication of this paper.

\section{Authors' Contributions}

Huiling Zhou and Jingjing Bao contributed equally to this work.

\section{Acknowledgments}

This work was supported by the funds from Taizhou social development project Foundation, Jiangsu, China (grant No. TS201625), the peak of Six Talents Project Foundation, Jiangsu, China (grant No. 2016-WSW-148), and the 333 project of scientific research project foundation, Jiangsu, China (grant No. BRA2017174).

\section{References}

[1] L. A. Torre, F. Bray, R. L. Siegel, J. Ferlay, and J. LortetTieulent, "Global cancer statistics, 2012," CA: A Cancer Journal for Clinicians, vol. 65, no. 2, pp. 87-108, 2015.
[2] J. D. Yang and L. R. Roberts, "Hepatocellular carcinoma: a global view," Nature Reviews Gastroenterology \& Hepatology, vol. 7, no. 8, pp. 448-458, 2010.

[3] E. A. Tsochatzis, T. Meyer, and A. K. Burroughs, "Hepatocellular Carcinoma," The New England Journal of Medicine, vol. 366, no. 1, p. 92; author reply 92-93, 2012.

[4] M. Frau, F. Biasi, F. Feo, and R. M. Pascale, "Prognostic markers and putative therapeutic targets for hepatocellular carcinoma," Molecular Aspects of Medicine, vol. 31, no. 2, pp. 179-193, 2010.

[5] B. L. Gallie, C. Campbell, H. Devlin, A. Duckett, and J. A. Squire, "Developmental basis of retinal-specific induction of cancer by RB mutation," Cancer Research, vol. 59, no. Suppl. 7, pp. 1731s1735s, 1999.

[6] M. Saijo, Y. Sakai, T. Kishino et al., "Molecular cloning of a human protein that binds to the retinoblastoma protein and chromosomal mapping," Genomics, vol. 27, no. 3, pp. 511-519, 1995.

[7] D. K. Dimova, O. Stevaux, M. V. Frolov, and N. J. Dyson, "Cell cycle-dependent and cell cycle-independent control of transcription by the Drosophila E2F/RB pathway," Genes \& Development, vol. 17, no. 18, pp. 2308-2320, 2003.

[8] M. B. Beasley, S. Lantuejoul, S. Abbondanzo et al., "The $\mathrm{P} 16 /$ cyclin $\mathrm{D} 1 / \mathrm{Rb}$ pathway in neuroendocrine tumors of the lung," Human Pathology, vol. 34, no. 2, pp. 136-142, 2003.

[9] M. J. Riemenschneider, R. Büschges, M. Wolter et al., "Amplification and overexpression of the MDM4 (MDMX) gene from 1q32 in a subset of malignant gliomas without TP53 mutation or MDM2 amplification," Cancer Research, vol. 59, no. 24, pp. 6091-6096, 1999.

[10] C. Zhao, W. Chen, S. Dai et al., "Expression and clinical role of RBQ3 in gliomas," Journal of the Neurological Sciences, vol. 359, no. 1-2, pp. 177-184, 2015.

[11] X. Zhao, J. Su, F. Wang et al., "Crosstalk between NSL Histone Acetyltransferase and MLL/SET Complexes: NSL Complex Functions in Promoting Histone H3K4 Di-Methylation Activity by MLL/SET Complexes," PLoS Genetics, vol. 9, no. 11, p. e1003940, 2013.

[12] H. Liu, E. H.-Y. Cheng, and J. J.-D. Hsieh, "Bimodal degradation of MLL by SCFSkp2 and APCCdc20 assures cell cycle execution: A critical regulatory circuit lost in leukemogenic MLL fusions," Genes \& Development, vol. 21, no. 19, pp. 2385-2398, 2007.

[13] D. G. Huntsman, S.-F. Chin, M. Muleris et al., "MLL2, the second human homolog of the Drosophila trithorax gene, maps to 19q13.1 and is amplified in solid tumor cell lines," Oncogene, vol. 18, no. 56, pp. 7975-7984, 1999.

[14] J. L. Hess, "MLL: A histone methyltransferase disrupted in leukemia," Trends in Molecular Medicine, vol. 10, no. 10, pp. 500507, 2004.

[15] S. Zhang, W. Shi, Y. Chen et al., "Overexpression of SYF2 correlates with enhanced cell growth and poor prognosis in human hepatocellular carcinoma," Molecular and Cellular Biochemistry, vol. 410, no. 1-2, pp. 1-9, 2015.

[16] J. T. Woitach, M. Zhang, C.-H. Niu, and S. S. Thorgeirsson, "A retinoblastoma-binding protein that affects cell-cycle control and confers transforming ability," Nature Genetics, vol. 19, no. 4, pp. 371-374, 1998.

[17] X. Zhang, C. He, C. He et al., "Nuclear PKM2 expression predicts poor prognosis in patients with esophageal squamous cell carcinoma," Pathology - Research and Practice, vol. 209, no. 8, pp. 510-515, 2013. 
[18] S. F. Altekruse, S. J. Henley, J. E. Cucinelli, and K. A. McGlynn, "Changing hepatocellular carcinoma incidence and liver cancer mortality rates in the United States," American Journal of Gastroenterology, vol. 109, no. 4, pp. 542-553, 2014.

[19] H. Liu, L. Ding, Y. Shen, F. zhong, Q. Wang, and X. Xu, "RBQ3 participates in multiple myeloma cell proliferation, adhesion and chemoresistance," International Journal of Biological Macromolecules, vol. 91, pp. 115-122, 2016.

[20] J. Wysocka, T. Swigut, T. A. Milne et al., "WDR5 associates with histone $\mathrm{H} 3$ methylated at $\mathrm{K} 4$ and is essential for H3 K4 methylation and vertebrate development," Cell, vol. 121, no. 6, pp. 859-872, 2005.

[21] Y. Dou, T. A. Milne, A. J. Ruthenburg et al., "Regulation of MLL1 H3K4 methyltransferase activity by its core components," Nature Structural \& Molecular Biology, vol. 13, no. 8, pp. 713-719, 2006.

[22] A. Mittal, F. Hobor, Y. Zhang et al., "The structure of the RbBP5 $\beta$-propeller domain reveals a surface with potential nucleic acid binding sites," Nucleic Acids Research, vol. 46, no. 7, pp. 38023812, 2018.

[23] P. Wang, C. Lin, E. R. Smith et al., "Global analysis of H3K4 methylation defines MLL family member targets and points to a role for MLL1-mediated H3K4 methylation in the regulation of transcriptional initiation by RNA polymerase II," Molecular and Cellular Biology, vol. 29, no. 22, pp. 6074-6085, 2009.

[24] S. Weirich, S. Kudithipudi, and A. Jeltsch, "Somatic cancer mutations in the MLL1 histone methyltransferase modulate its enzymatic activity and dependence on the WDR5/RBBP5/ASH2L complex," Molecular Oncology, vol. 11, no. 4, pp. 373-387, 2017.

[25] A. Ali, S. N. Veeranki, and S. Tyagi, "A SET-domainindependent role of WRAD complex in cell-cycle regulatory function of mixed lineage leukemia," Nucleic Acids Research, vol. 42, no. 12, pp. 7611-7624, 2014. 


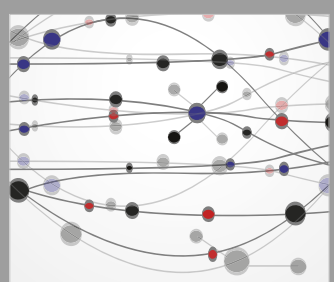

The Scientific World Journal
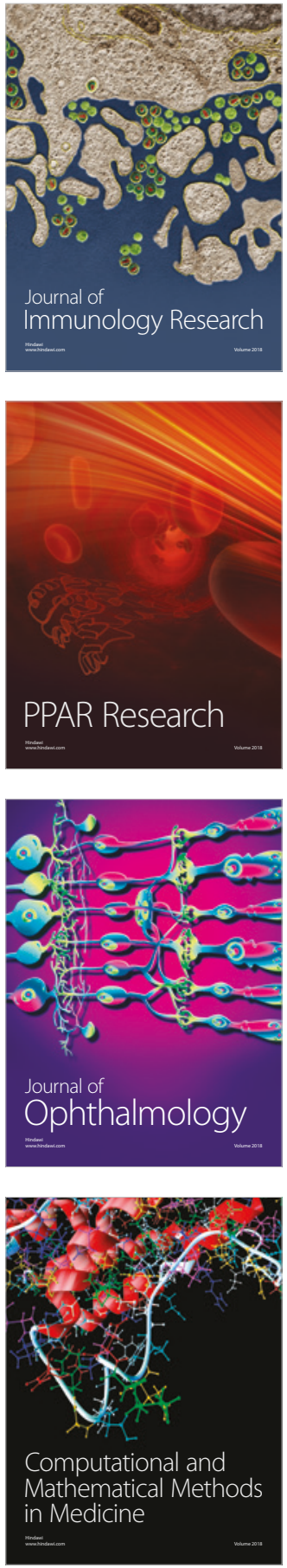

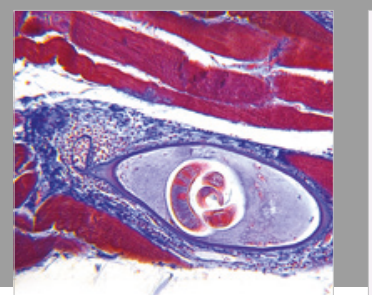

Gastroenterology Research and Practice

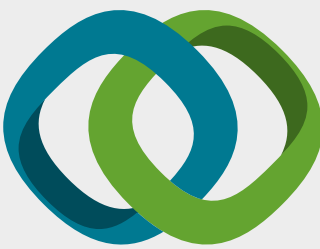

\section{Hindawi}

Submit your manuscripts at

www.hindawi.com
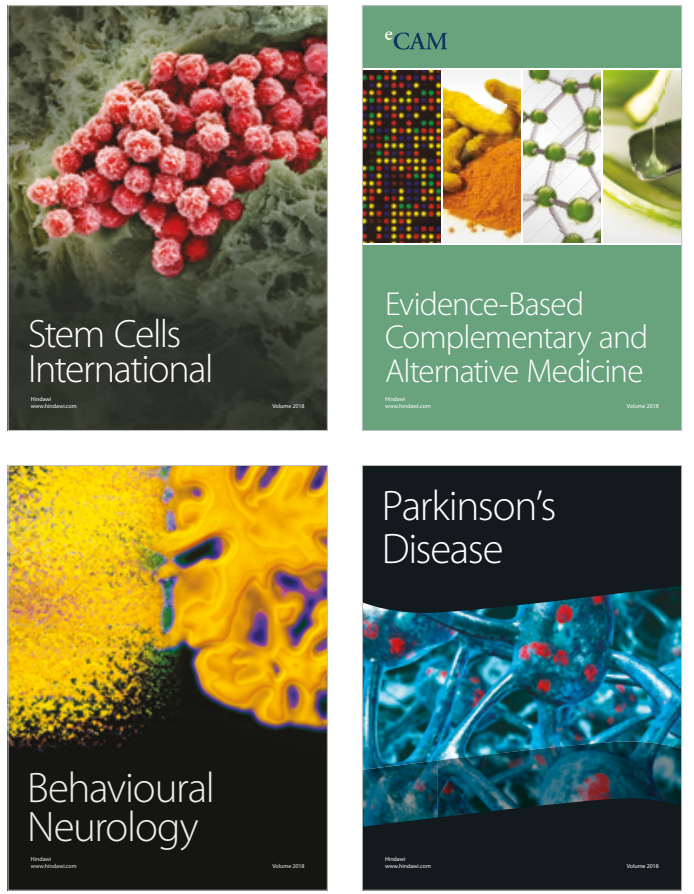

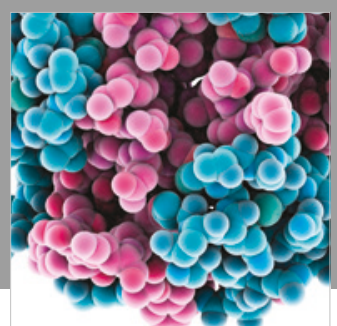

ournal of

Diabetes Research

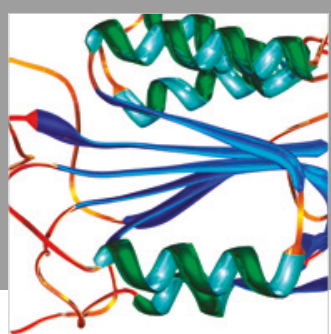

Disease Markers
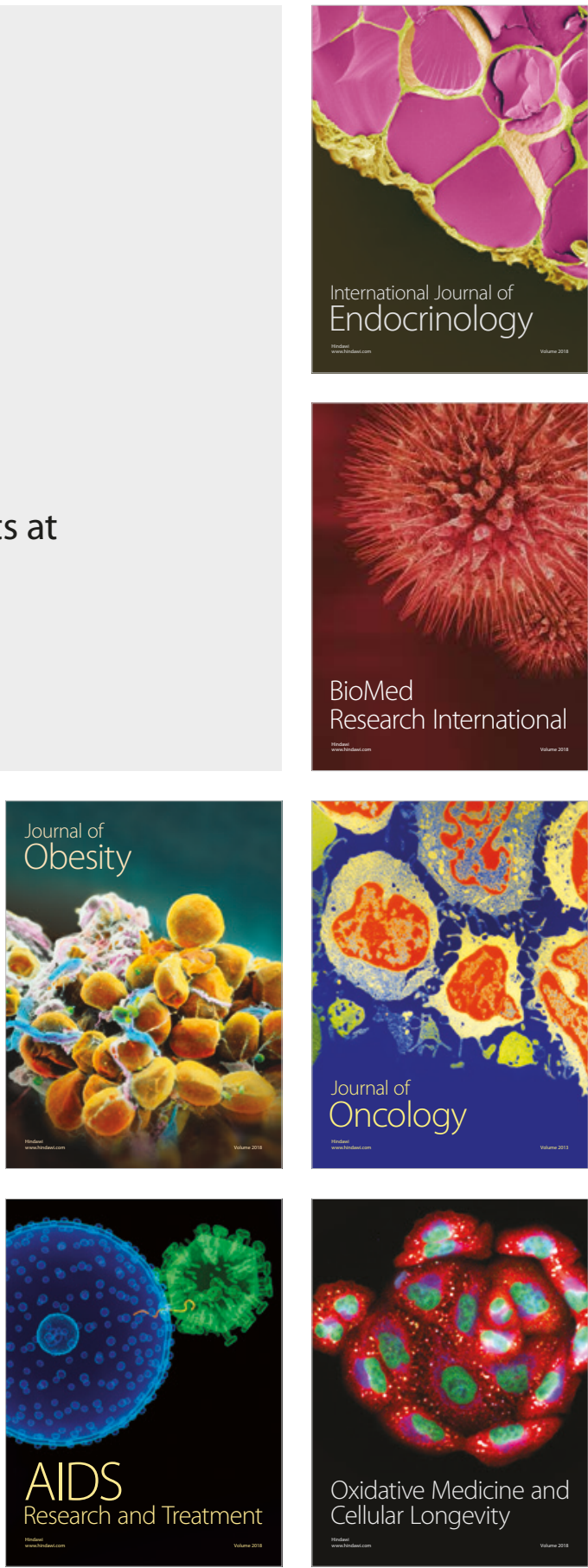